\title{
Plasma Rico en Plaquetas y su utilización en implantología dental
}

\author{
GARCÍA GARCÍA V * \\ CORRAL I ** \\ BASCONES MARTÍNEZ A ***
}

\begin{abstract}
García García V, Corral I, Bascones Martínez A. Plasma Rico en Plaquetas y su utilización en implantología dental. Av Periodon Implantol. 2004; 16, 2: 81-92.
\end{abstract}

\begin{abstract}
RESUMEN
Se presenta una revisión sobre la utilización del Plasma Rico en Plaquetas en el ámbito de la implantología. El trabajo comienza con la definición de varios conceptos básicos sobre Regeneración Tisular Guiada. A continuación se explican los factores de crecimiento y su participación en los concentrados de plaquetas. Se hace hincapié en los estudios realizados con PRP e implantes y se describe la aparición de unos posibles efectos negativos con el empleo de esta técnica. Por último se exponen las conclusiones del trabajo.
\end{abstract}

\section{PALABRAS CLAVE}

Plasma Rico en Plaquetas, PRP, factores de crecimiento, regeneración ósea.

Aceptado para publicación: Junio 2003

\section{INTRODUCCIÓN}

En la década de los '80 el cirujano, a la hora de insertar los implantes dentales, tenía como principal preocupación que la cantidad de hueso residual fuera cuantitativa y cualitativamente suficiente para poder mantenerlos a lo largo del tiempo. Hoy día, gracias a la aparición de las técnicas de Regeneración ósea Guiada, y dentro de estas la utilización del Plasma Rico en Plaquetas (PRP), se van a poder colocar implantes en localizaciones donde años antes hubiera sido impensable. Cada vez son más los clínicos que se decantan por la utilización de los concentrados de plaquetas, siendo este auge en su demanda debido a la aparición de técnicas simplificadas que permiten una aplicación a nivel ambulatorio.

No obstante, comienzan a publicarse artículos mostrando unos posibles efectos negativos derivados de la utilización del PRP. De momento, son hipótesis que deben confirmarse con más estudios clínicos.

\section{CONCEPTOS GENERALES}

Ante una agresión que supone una pérdida de tejido, el organismo responde con un proceso de restauración del tejido afectado. Dicho proceso se inicia con la aparición de un coágulo sanguíneo, que va diferenciándose en un tejido fibroso, el cuál rellena el defecto. Este tejido dañado no conserva ni su arquitectura ni su función original, y sus propiedades y características no se corresponden con las que previamente existían; en este caso se ha producido una reparación del tejido (l).

En algunos casos el proceso de restauración tiende hacia la creación de un tejido similar al original y no hay diferenciación alguna con el tejido circundante: en este caso se habla de regeneración del tejido (Robin) (1). (Figura 1).

Clásicamente se admite que en presencia de un defecto óseo reciente se origina un crecimiento para-

*** Catedrático en Medicina Bucal y Periodoncia. Universida Complutense de Madrid. 


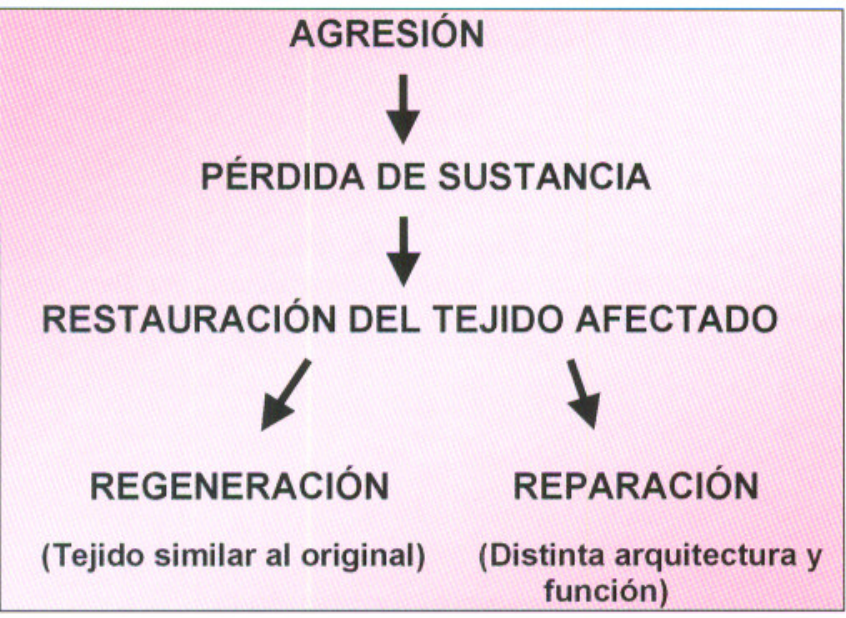

Fig 1: Proceso de restauración del tejido afectado.

lelo y competitivo entre el tejido óseo remanente y los tejidos blandos adyacentes para ocuparlo. El resultado en la mayoría de los casos es la aparición de un defecto permanente cubierto por tejido gingival (2).

Las técnicas de Regeneración Tisular Guiada (RTG) se fundamentan en base al principio de exclusión celular, en el que se demostraba cómo las células de cada línea podían proliferar de forma independiente (Gottlow) . Su meta principal es obtener la regeneración de los tejidos, evitando la reparación de los mismos $(1,3)$.

Existen tres mecanismos relacionados con la regeneración del tejido óseo:

1. Osteogénesis: es la creación de hueso nuevo a cargo de las células competentes, en este caso los osteoblastos, cuya fuente son los injertos óseos autó$\log$ os (1).

2. Osteoconducción: es la capacidad de servir de guía para el crecimiento óseo y permitir el depósito de hueso nuevo, aislando el defecto e impidiendo el crecimiento de tejido conjuntivo hacia el interior del mismo $(1,2)$.

3. Osteoinducción: consiste en la producción de señales reguladoras del metabolismo óseo. Dentro de esta vertiente englobamos a las proteínas morfogenéticas óseas (BMP), que actúan directamente sobre las células precursoras promoviendo la formación de hueso nuevo en cantidades ilimitadas; $y$ a los factores de crecimiento (FC), que modifican la proporción de hueso preexistente, aumentan las mitosis y la secreción de proteínas de las células presentes, confiriendo a las células óseas una limitada capacidad de regeneración $(1,4)$.

El hueso es un tejido vivo que presenta un continuo recambio celular mediado por las células óseas (oste- oblastos y osteoclastos), formando las unidades de recambio óseo (4).

En la remodelación ósea influye una serie de factores humorales que actuarán a nivel sistémico como la hormona paratiroidea (PTH), segregada por la glándula paratiroides, y que controla los niveles de calcio y fósforo en la sangre; la vitamina D y la calcitonina (4).

Entre los factores que influyen en el remodelado a nivel local están: los FC, las BMP, las citoquinas, las cininas y las prostaglandinas 4 .

\section{FACTORES DE CRECIMIENTO}

Los factores de crecimiento son una familia de señales peptídicas moleculares capaces de modificar las respuestas biológicas celulares, estando sobretodo involucradas en el control del crecimiento y diferenciación celular. Son mediadores biológicos que regulan la migración, proliferación, diferenciación y metabolismo celular $(5,4)$.

En cuanto a su clasificación, los FC se pueden clasificar según sea su especialidad: amplia o reducida. Los de especialidad amplia como el PDGF y el EGF actúan sobre muchas clases de células ,entre ellas tenemos: fibroblastos, fibras musculares lisas, células neurogliales y el último, además, sobre células epiteliales y no epiteliales.

Como ejemplo de FC de especificidad reducida tenemos a la eritropoyetina que sólo induce la proliferación de los precursores de los hematíes (6).

Los factores de crecimiento actúan de manera local. La estimulación celular se realiza bien por un sistema autocrino, es decir, las células producen y responden al mediador biológico, o por un sistema paracrino en el que la célula que produce el factor se encuentra en las proximidades de las células a las que afecta $(5,4)$.

En general, los factores de crecimiento son sintetizados en forma de precursores, siendo necesario para la liberación del factor en forma "activa" un proceso específico de proteolisis $(5,4)$.

Su mecanismo de acción siempre comienza al unirse a receptores específicos de membrana (5). Para cada clase de FC existe un receptor o conjunto de receptores específicos. Las células responden a un FC sólo si disponen de la proteína receptora apropiada (6). Los factores son el estímulo necesario para iniciar una cadena de eventos celulares que tienen como resultado las funciones anteriormente mencionadas. El pro- 
ceso está mediado por un sistema de segundos mensajeros en el que interviene una proteína tirosínquinasa . Debido a este mecanismo, la acción de los factores en el lugar de la lesión continúa aunque hayan desaparecido los mismos del medio, ya que han activado el sistema de segundos mensajeros $(5,4)$.

Entre los tipos celulares productores de los factores de crecimiento están los fibroblastos, osteoblastos, células endoteliales y leucocitos, especialmente, monocitos y macrófagos. Además existen lugares de almacenamiento, como son las plaquetas (en los gránulos $\alpha$ ) y el hueso (adheridos a la matriz ósea) $(5,4)$.

Los mecanismos reparativos y la liberación de los factores de crecimiento óseo son activados ante cualquier noxa que altere la morfología estructural o celular del tejido óseo. Se incluyen como desencadenantes de activación de los factores: el traumatismo, accidental o quirúrgico, del tejido óseo como la pérdida dental o la colocación de implantes, la interrupción temporal del aporte vascular asociado a la desvitalización y necrosis del tejido óseo, e incluso las alteraciones humorales con repercusiones en el metabolismo del calcio (8).

\section{PLASIMA RICO EN PLAQUETAS}

El estudio de los factores de crecimiento junto con el descubrimiento de su liberación por parte de las plaquetas ha conducido al desarrollo de un concentrado de plaquetas autólogo, ideal para mejorar el proceso de cicatrización de los tejidos blandos y la regeneración ósea $(9,10)$.

El PRP (Plasma Rico en Plaquetas) se define como el contenido en plaquetas en forma de sobrenadante tras la centrifugación de sangre anticoagulada (10).

Las plaquetas desempeñan un papel muy importante dentro del PRP, ya que constituyen la principal fuente de actividad mitógena en el plasma sanguíneo (11) y van a funcionar como vehículo portador de factores de crecimiento y de otras proteínas que desempeñan un papel importante en la biología ósea, como son la fibronectina y otras proteínas adhesivas (7).

Los principales FC contenidos en el Plasma Rico en Plaquetas son los siguientes:

\section{- Factor de Crecimiento Derivado de las Plaquetas (PDGF)}

Se le denominó de esta forma por encontrarse por vez primera en las plaquetas, donde se almacena dentro

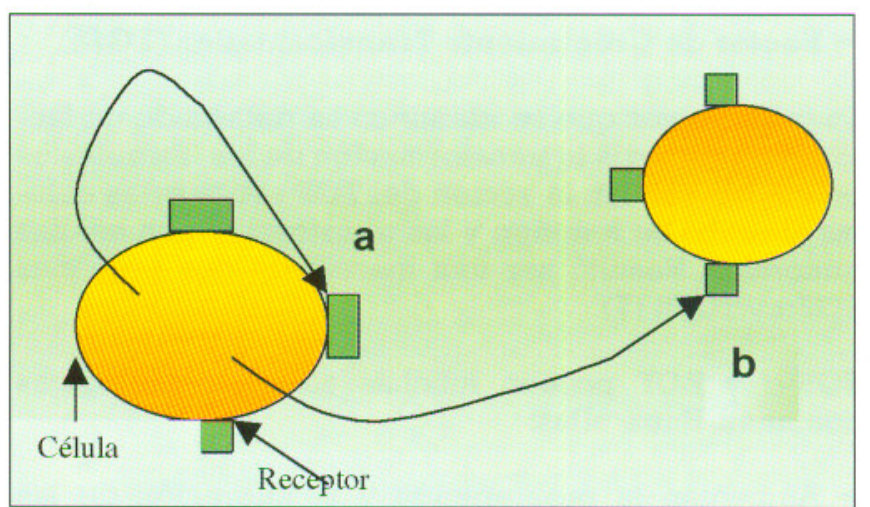

Fig 2: a: GF actúa de forma autocrina, es decir, interacciona con los autorreceptores de la misma célula que lo sintetiza. b: GF actúa como factor de crecimiento paracrino, esto es, ejerce su acción en otra célula adyacente o distante (7)

de los gránulos alfa, pero también es producido por otros tipos celulares como son los macrófagos, células endoteliales, monocitos, fibroblastos, hallándose también en la matriz ósea $(5,12)$.

Según las cadenas que formen la estructura del factor de crecimiento, nos encontramos con tres formas (5, 1): PDGF-AA, PDGF-BB y PDGF-AB.

Entre sus acciones biológicas podemos destacar:

- Participación en la glucogénesis (5).

- Regulación del crecimiento y diferenciación celular en el sistema nervioso central durante su desarrollo5.

- Favorece la cicatrización. Su función en la reparación tisular se basa en inducir la mitogénesis (aumentar el número de células para la cicatrización), estimular la angiogénesis de la mitosis de células endoteliales (lo cual se traduce en la formación de yemas capilares), producción de proteínas de la matriz extracelular y en la quimiotaxis de fibroblastos, monocitos, células musculares y macrófagos. También estimula la fagocitosis en los neutrófilos y monocitos $(5,14,13,9,15)$.

- Estimula la producción de fibronectina, una molécula de adhesión celular utilizada durante la proliferación y migración celular en la cicatrización; y ácido hialurónico (9).

- Se ha involucrado en el desarrollo de agrandamientos gingivales tras la toma de fenitoína. La fenitoína aumentaba la producción de PDGF por los macrófagos y la excesiva producción de este factor en la encía provocaba su hipertrofia (5).

- Puede estimular las somatomedinas (5).

Aumenta la regeneración periodontal (5). 


\section{> Factor de Crecimiento Transformador (TGF)}

La primera vez que se identificó se trataba de un factor que promovía la transformación de los fibroblastos en cultivo celular, la acción del TGF sobre estas células alteraba su fenotipo y las transformaba en células tumorales. Resultó ser una mezcla de dos proteínas TGF $\alpha$ y TGF $\beta$ (7).

TGF- $\alpha$ y EGF poseen muchas acciones biológicas comunes. Entre ellas:

- Aumentan la proliferación y la migración de las células epiteliales.

> Liberan iones calcio del hueso. El TGF- $\alpha$ es de 3 a 10 veces más potente que el EGF.

> Inhibe la actividad de los osteoblastos. De 10 a 100 veces más potente que el TGF- $\alpha$.

$>$ Tiene efecto angiogénico.

$>$ Interviene en el desarrollo tumoral por dos mecanismos:

- Estimulando la proliferación celular por un mecanismo autocrino.

- Induciendo la angiogénesis tumoral usando un mecanismo paracrino (5).

Las acciones biológicas del TGF-b quedan resumidas en la figura 3.

\section{> Factor de Crecimiento Epidérmico(EGF)}

EGF es sintetizado como un precursor de 1217 aminoácidos (cuyo peso molecular es 133.000) que incluye 8 secuencias de aminoácidos homólogas al factor de crecimiento. Los fibroblastos del ligamento periodontal, los preosteoblastos y precondrocitos expresan un alto número de receptores para el EGF (5).

Entre sus acciones biológicas podemos destacar:

- Efectos mitogénicos y quimiotácticos en fibroblastos y células epiteliales (5). También induce la migración celular (15), y se ha demostrado que tiene un efecto dosis-dependiente (1).

- Induce la formación rápida del diente. Thesleff en 1987 demostró la presencia de receptores de EGF en los tejidos apicales de dientes en erupción.

- Estimula la formación del tejido de granulación.

- Inhibe la liberación de ácido por la mucosa gástrica (5).

- Aunque el EGF no aumenta la síntesis de RNA mensajero para proteínas de la matriz extracelular como el colágeno, los trabajos recientes apuntan a que lo hace por un mecanismo indirecto, atrayendo fibroblastos por quimiotaxis, estos a su vez sintetizan colágeno produciéndose un aumento del colágeno total (7).

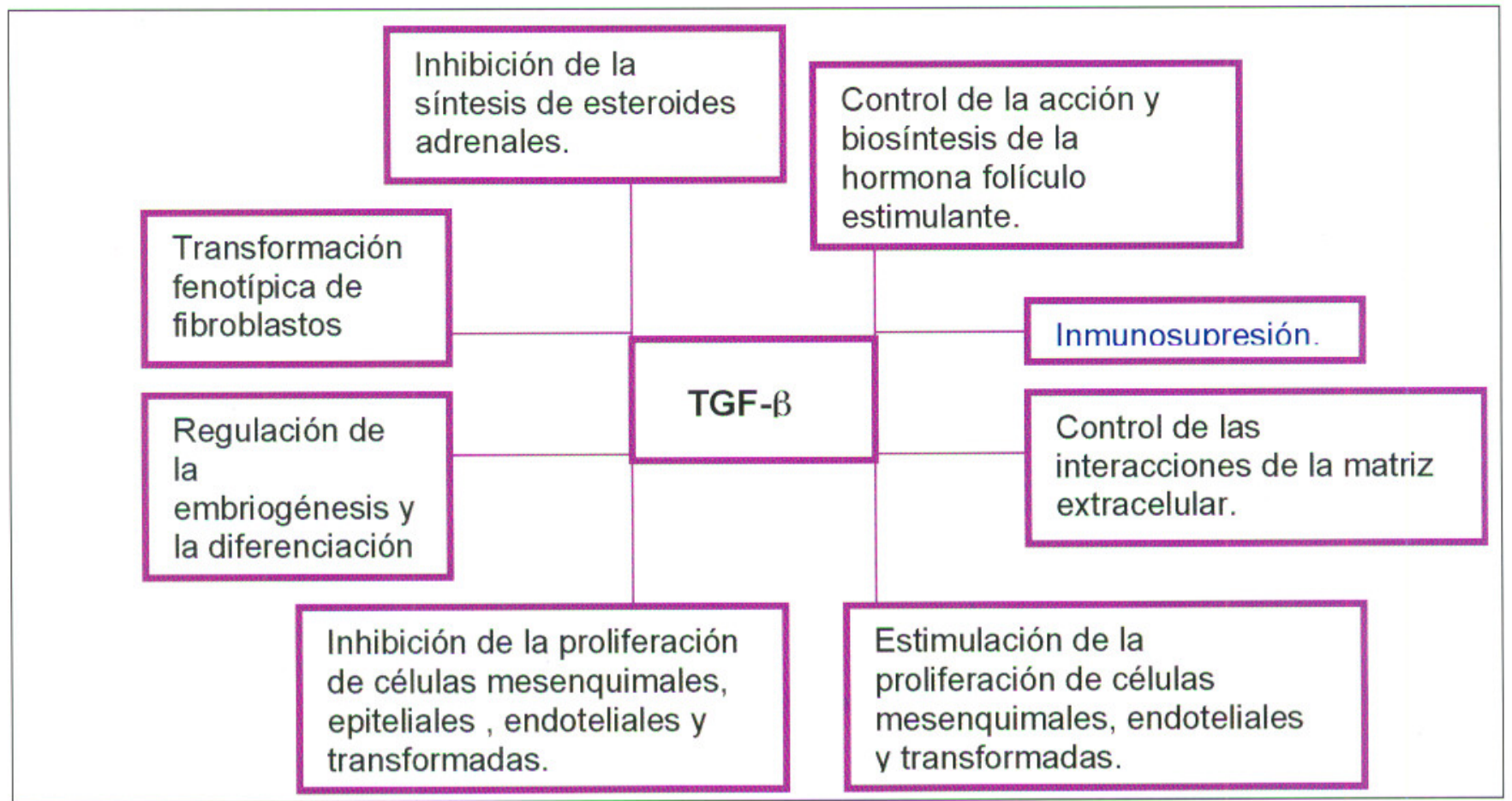

Fig. 3: Principales acciones biológicas del TGF- $\beta$ (5). 


\section{$>$ Factor de Crecimiento Fibroblástico (FGF)}

Son una familia de polipéptidos cuya misión es la de controlar la proliferación, diferenciación y otras funciones celulares en aquellas células derivadas del mesodermo y neuroectodermo. Existen dos tipos: FGF ácido y FGF básico (5).

Entre sus acciones biológicas están las siguientes:

- Estimulación de la angiogénesis por un mecanismo directo, al estimular la mitosis y migración de las células endoteliales (4).

- Estimulación y coordinación de la mitogénesis de múltiples tipos celulares como células de origen mesenquimatoso, como los fibroblastos, los osteoblastos, condorcitos, células musculares lisas y mioblastos esqueléticos durante el crecimiento animal, mantenimiento y reparación tisular (4).

\section{- Factor de Crecimiento semejante a la insulina (IGF)}

Es una familia de proteínas séricas con una estructura en cadena simple que poseen una semejanza del $50 \%$ con la insulina. Existen dos tipos: IGF-I e IGF-II (5). Se ha demostrado que el activo a nivel de crecimiento óseo es el IGF-I (1). En el hueso se sintetizan altos niveles de IGF-I y es secretado por los osteoblastos, IGF-I regula por tanto la formación de hueso de forma autocrina y también aumenta el número de células multinucleadas osteoclásticas (7).

Entre sus acciones biológicas podemos destacar las siguientes:

- Su capacidad de estimular la síntesis de matriz ósea por (5):

- Un efecto directo en la función diferenciadora de los osteoblastos.

- Un aumento en la replicación de las células osteoprogenitoras.

- Es capaz de estimular la actividad mitogénica y actúa como factor quimiotáctico de las células del ligamento periodontal (5).

- Es un agente quimiotáctico potente para las células vasculares endoteliales, originando un aumento de neovascularización de la herida (15).

- IGF-I estimula la síntesis de glucógeno en el hígado (5).

- Es capaz de actuar de forma sinérgica con el PDGF aumentando la regeneración periodontal (16).

\section{> Factor de Crecimiento Vascular Endotelial (VGF)}

Se aisló originalmente a partir de cultivos celulares de hipófisis. Se trata de una proteína homodimérica cuya secuencia de aminoácidos tiene una similitud del $24 \%$ con PDGF- $\beta$, pero se une a diferentes receptores que el PDGF e induce distintos efectos biológicos (7).

Es un mitógeno potente y selectivo para las células endoteliales. Aunque no se conoce con detalle su papel en la regeneración, su importancia queda manifiesta por su acción angiogénica in vivo $(15,1,7)$.

En un estudio realizado por Roberto Corneliniy cols., se demuetra que el VEGF es un factor implicado tanto en el mantenimiento de la fisiología periodontal como en la progresión de periimplantitis, aunque todavía se requieren más estudios para aclarar su papel en estos procesos (17).

\section{- Factor de crecimiento Derivado del Cemento (CGF)}

En 1993, Narayanan y Yonemura aislaron una nueva especie de factor de crecimiento en el cemento que no se asemeja en sus características a ninguno de los conocidos.

Es mitógeno para los fibroblastos gingivales del ligamento periodontal y dérmico. Su acción como mitógeno está potenciada por el EGF. No se conoce todavía como se libera ni el probable potencial del cemento para regular el metabolismo y recambio de los tejidos de alrededor (5).

Meraw y cols. realizaron un experimento para examinar los efectos del CGF en la reparación de defectos óseos periimplantarios resultando el uso de este factor en un aumento de hueso en la interfase huesoimplante y en la cantidad de hueso periférico (8).

\section{> Factor Plaquetario 4 (FP-4)}

Se trata de un factor de crecimiento quimiotáctico para los neutrófilos que es también liberado de los gránulos-a plaquetarios. Así pues, parece ser el responsable de la afluencia de neutrófilos en el proceso de cicatrización (18).

Se han realizado experimentos para descubrir los efectos beneficiosos de combinar varios factores de crecimiento:

- El uso combinado de factores de crecimiento ha mostrado unos efectos sinérgicos para la mejora en la cicatrización. Esto se ha visto al combinar PDGF con IGF, PDGF con TGF- $\alpha(8,19)$. 
o Al utilizar conjuntamente in vitro BMP-2, TGF- $\beta$, FGFb y PDGF se ha obtenido una mejora en la respuesta mitógena de los fibroblastos dérmicos y de los osteoclastos (8).

o Mustoe et al. Comprobaron que al combinar PDGF con TGF- $\beta$ se producía un aumento significativo del tejido de granulación (9).

o Los factores que tienen un efecto beneficioso en la regeneración periodontal tanto aislados como en combinación son: PDGF, IGF, FGF y TGF- $\beta$ (13). Lynch y cols. comprobaron un aumento en la regeneración periodontal al utilizar en combinación PDGF y IGF-I $(20,5,21)$.

o Al utilizar conjuntamente IGF-I y PDGF-B en cultivos de rata se puede apreciar un aumento en la deposición de matriz ósea más que si se utilizara cada factor por separado (22).

Con el PRP se pretende actuar en la primera etapa de la reparación y sustituir el coágulo de sangre que se formaría, rellenando en su lugar el alvéolo con un coágulo de PRP. Así, al mismo tiempo que sellamos el alvéolo evitando su contaminación proporcionamos una gran concentración de factores de crecimiento y otras proteínas adhesivas que aceleran y optimizan las primeras etapas del proceso regenerativo del hueso (3).

\section{MECANISMO DE ACCIÓN DEL PRP}

Para ver la acción del PRP vamos a exponer un modelo de regeneración ósea simplificado utilizando un injerto particulado de hueso esponjoso y medular junto con PRP:

1. Los tejidos de la zona con déficit óseo se encuentran en un ambiente hipóxico y ácido conteniendo plaquetas, leucocitos, hematíes y fibrina en un complejo coágulo adyacente a los osteocitos, osteoblastos y células madre que han sido transferidas 19. Un coágulo hemático natural contiene $95 \%$ de hematíes, $5 \%$ de plaquetas y menos de $1 \%$ de leucocitos en una malla de numerosos filamentos de fibrina. Un coágulo rico en plaquetas contiene $4 \%$ de células rojas, $95 \%$ de plaquetas y $1 \%$ de leucocitos (4).

A nivel exterior del periostio donde el cirujano realizó la sutura, los tejidos son fisiológicamente normales, es decir con unos niveles de oxígeno y un Ph fisiológicos. Contienen una población de células estructurales, células madre relacionadas con la cicatrización y capilares seccionados con coágulos y células endoteliales expuestas (13) (Fig.4).

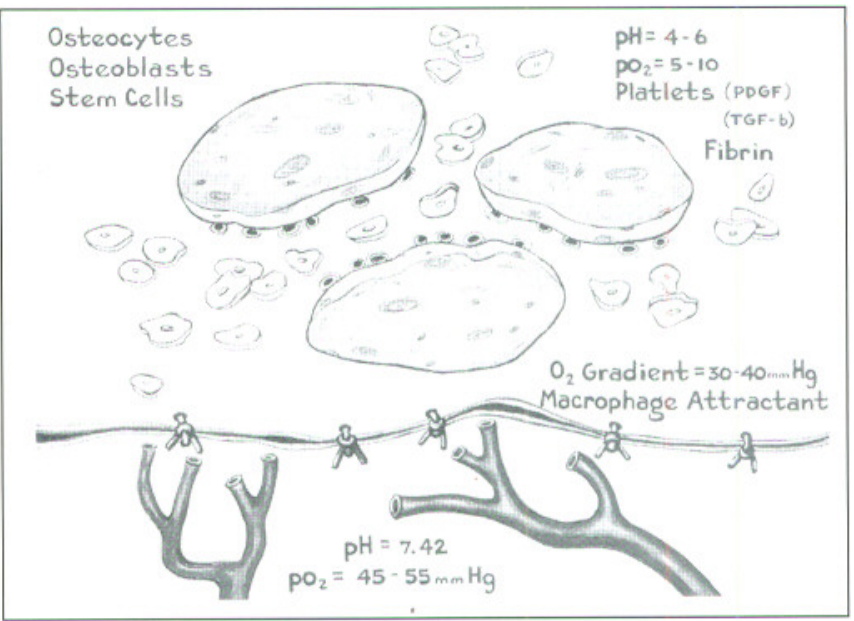

Fig. 4: Células básicas, bioquímica y factores de crecimiento asociados dentro y fuera del espacio ocupado por el injerto (13). Imagen tomada del artículo de Marx y colaboradores.

2. Las plaquetas son fragmentos anucleares de los megacariocitos, con una forma discoide y cuya cantidad normal en sangre se ha considerado habitualmente de 150.000-400.000/ $\mu$ l. Igual que cuando se produce una herida, a la membrana plaquetaria se une el factor plasmático de Von Willebrand (a través de la glicoproteína Ib) que hace que se unan al colágeno expuesto de la pared vascular (adhesión), y de esta manera se unan entre sí (agregación). La activación de las plaquetas (degranulación) se realiza por la adhesión de las mismas al colágeno y otros componentes del subendotelio, o por la presencia de trombina (23).

El inicio de la regeneración ósea comienza con la liberación en el injerto de PDGF y TGF- $\beta$ a partir de la degranulación plaquetaria. El PDGF estimula la mitogénesis de las células madre medulares y de los osteoblastos transferidos en el injerto aumentando su número. También comienza la angiogénesis a nivel de los capilares mediante la inducción de mitosis en las células endoteliales (13).

El TGF- $\beta$ inicialmente activa a los fibroblastos y preosteoblastos induciendo su mitosis para aumentar el número de los mismos, así como para promover su diferenciación hacia osteoblastos funcionales maduros. La secreción continuada de TGF- $\beta$ induce a los osteoblastos para que depositen matriz ósea y a los fibroblastos para depositar matriz colágena que sustente el crecimiento capilarl3.(Fig.5)

La vida media de las plaquetas en la zona de cicatrización y el periodo de influencia directa de sus factores de crecimiento es menor a 5 días. La continuación de la cicatrización y regeneración ósea tiene lugar por dos mecanismos: 


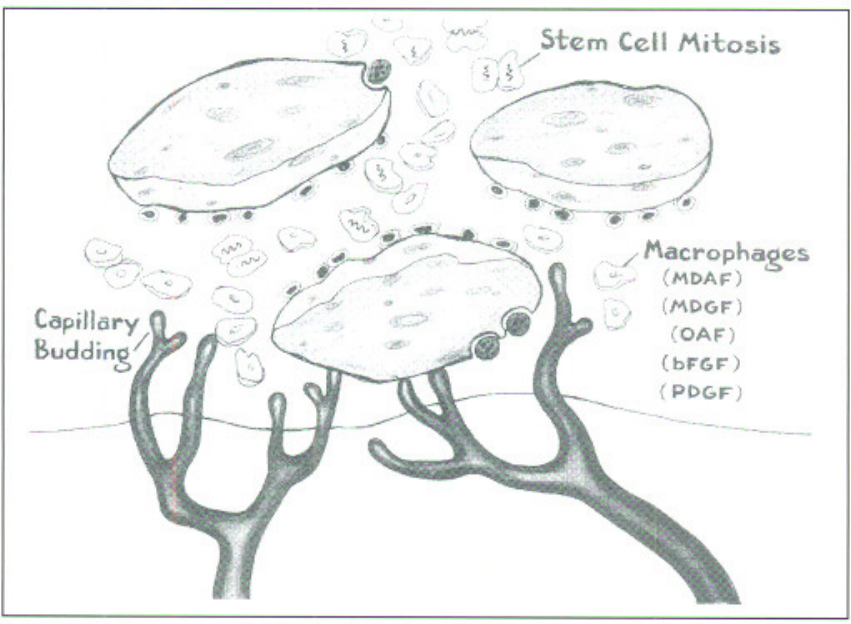

Fig. 5: Al tercer día comienza el crecimiento de los capilares sanguíneos en respuesta a la acción del PDGF y TGF- $\beta$. Los macrófagos se convierten en las principales células productoras de macrófagos, ya que las plaquetas en este período de tiempo han completado la degranulación (13). Imagen tomada del artículo de Marx y colaboradores.

$\checkmark$ La formación de TGF- $\beta$ por parte de los osteoblastos recién formados.

$\checkmark$ La quimiotaxis y activación de los macrófagos que sustituyen a las plaquetas como principal fuente de factores de crecimiento hacia el tercer día (13).

\section{TÉCNICAS PARA LA OBTENCIÓN DEL PRP}

En respuesta a la necesidad de mejorar los agentes hemostáticos y los adhesivos quirúrgicos se desarrolló en los años 80 la cola de fibrina o adhesivo de fibrina siendo comercializada con el nombre de Tisucol ${ }^{\oplus}$. En EEUU se prohibió su utilización por riesgos potenciales de infección por transmisión vírica, hepatitis $\mathrm{C}$ y SIDA entre otros (7).
Tayapongsak y cols. obtuvieron un adhesivo de fibrina autóloga con propiedades hemostáticas que reducía el tiempo de remodelación a la mitad cuando se utilizaba en injertos de hueso medular y esponjoso (24).

Después se desarrolla la técnica de obtención del gel de plaquetas que se diferencia del adhesivo de fibrina en que en el gel de plaquetas estaban presentes todas las proteínas plaquetarias y la concentración de fibrinógeno es más reducida (7).

El éxito del gel de fibrina en cirugía ha llevado al desarrollo de una técnica con la misma filosofía, pero en otra escala de volúmenes de sangre que puede ser empleada rutinariamente en las consultas dentales y es denominada por Anitua como Agregado de Plaquetas (P.R.G.F) (7).

Esencialmente, la sangre completa es anticoagulada con citrato y entonces se centrifuga en sus tres componentes básicos por gradiente de densidades (4).

Existen técnicas de uno o dos centrifugados .Los kits PCCS y Curasan PRP son sistemas de dos centrifugaciones (23), los cuales trabajan como puede apreciarse en la figura $6(25,26)$.

El PRP se puede obtener en el quirófano o de modo ambulatorio en el gabinete dental:

\section{a) Sistema de quirófano:}

Cuando se realiza la intervención en quirófano, con anestesia general, y cirugía mayor, el separador celular saca 400 a $500 \mathrm{ml}$ de sangre autóloga completa, a una velocidad de $50 \mathrm{ml}$ por min .Para obtener $40 \mathrm{ml}$ de PRP serán necesarios $400 \mathrm{ml}$ de sangre completa $(18,4)$. El componente PPP es acelular, supone unos $200 \mathrm{ml}$ y retorna al paciente. El componente RBC fundamentalmente concentrado de hematíes supone alrededor de $180 \mathrm{ml}$ de volumen y también se retorna al paciente (4).

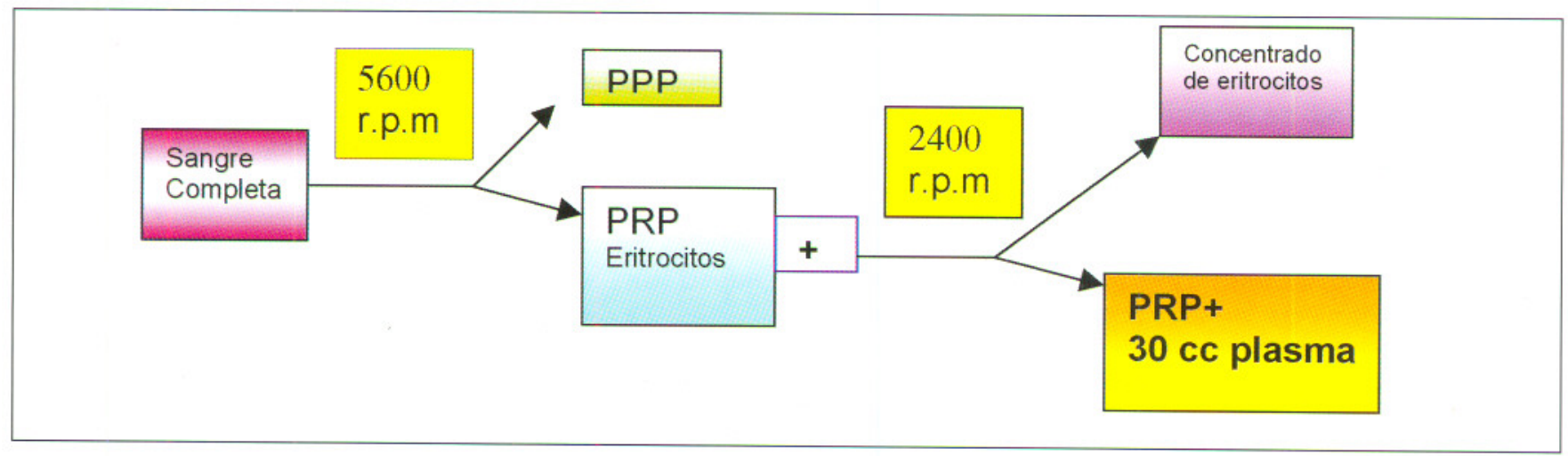

Fig. 6: Diferencias entre los sistemas de una y dos centrifugaciones. 


\section{b) Sistema de gabinete dental:}

Los avances tecnológicos han permitido reducir la extracción sanguínea necesaria para obtener el producto final de $450 \mathrm{ml}$ a $40-110 \mathrm{ml}$, una cantidad mucho más manejable $(4,25)$. Así se consigue de 6 a $12 \mathrm{ml}$ de PRP desechando la sangre no utilizada, ya que es innecesaria la re-infusión y está contraindicada su reutilización en el gabinete dental (4).

Actualmente hay varios aparatos para la obtención de PRP en la clínica dental, son la Smart PRP (Harvest Technologies, Norwell,MA), el 3i Platelet Concentrate Collection System, The Plasma Seal (Plasma Seal, San Francisco, CA), y el Platelet Concentrator (Impl Innovations, West Palm Beach, FL) ,entre otros. Todos pueden procesar cantidades pequeñas de sangre total, de 45 a $60 \mathrm{ml}$, para obtener de 5 a $6 \mathrm{ml}$ de PRP, por ciclo $(4,18)$.

El Smart PRP y el 3i Platelet Concentrate Collection System son los únicos, aceptados por la FDA para la producción de PRP $(8,18)$.

De manera sistemática el método de obtención de PRGF propuesto por el Dr.Anitua es el siguiente (7):

1. Se realiza la extracción de sangre unos minutos antes de comenzar la cirugía. Se utilizan tubos estériles con citrato sódico al 3,8\% como anticoagulante7. el hecho de realizar la extracción de sangre de forma previa y no utilizar sangre resultante de la cirugía es porque la cirugía , por sí misma, implica la activación de la cascada de la coagulación (28).

2. Se centrífuga el plasma con un equipo digital durante 7 minutos, a una velocidad de centrifugación de $280 \mathrm{G}$ a temperatura ambiente.

3. El plasma se separa en fracciones mediante pipeteado muy meticuloso para no crear turbulencias en las fracciones obtenidas. Si se remueve la serie roja se debe desechar el tubo contaminado debido a que se ha producido la hemolización del plasma.

Los primeros o,5 cc (fracción 1), es un plasma pobre en plaquetas y por tanto pobre en factores de crecimiento.Los siguientes o,5 cc (fracción 2) corresponderán a un plasma con un número de plaquetas similar al que tiene la sangre periférica.La fracción de plasma rico en plaquetas son los o,5 cc inmediatamente encima de la serie roja (fracción 3) (7).

4. Una vez que tenemos la fracción de PRP, para provocar la formación del coágulo añadimos $0,05 \mathrm{cc}$ de cloruro cálcico al $10 \%$ por cada cc de PRP y entre 5 y 8 minutos se nos formará el coágulo (7).
Se ha demostrado que las plaquetas más recientemente sintetizadas y, por lo tanto, con una gran actividad, son grandes y están mezcladas con el primer milímetro de la fracción de células rojas, por lo que algunos autores proponen el utilizar también esta pequeña parte (13).

Si cogemos las dos primeras fracciones y las añadimos también cloruro cálcico obtenemos fibrina autóloga que se puede utilizar como membrana o tapón hemostático, dado su alto poder cicatrizante (2).

\section{UTILIZACIÓN DEL PRP EN IMPLANTOLOGÍA}

En la literatura podemos encontrar experimentos realizados con PRP en los que los resultados obtenidos son beneficiosos:

Kim y cols. realizaron un experimento para observar las diferencias en cuanto a la reparación de defectos óseos alrededor de los implantes de titanio con el uso de hueso liofilizado (DBP) en un grupo de perros , hueso liofilizado + PRP en un segundo grupo y un tercer grupo control. Los resultados obtenidos fueron la aparición de hueso nuevo alrededor de los implantes tanto en el primer como en el segundo grupo y un mayor porcentaje de contacto hueso-implante en el grupo tratado con hueso liofilizado y PRP respecto a los otros dos grupos (29). En otro experimento comprobaron el efecto del Cemento Particulado Dentinario Paris en combinación con o sin PRP para el tratamiento de los defectos óseos alrededor de los implantes. En la histometría se apreciaba un mayor porcentaje de contacto óseo en los casos en los que se utilizó Cemento Particulado Dentinario Paris y PRP , en comparación con el grupo control y con los que sólo fueron tratados con el cemento (30).

Lynch y cols, en 1991, utilizaron PDGF-BB+IGF-I en un gel de metilcelulosa en perros Beagle y observaron que a los 7 días, el porcentaje de hueso que rellenaba el espacio peri-implantario y el porcentaje de superficie del implante en contacto con nuevo hueso eran significativos frente a los controles. A los 21 días, el porcentaje de hueso que rellenaba el espacio periimplantario seguía siendo significativo pero no el porcentaje de superficie del implante en contacto con el hueso (5).

Becker y cols, realizaron en 1992 un experimento con PDGF-BB+IGF-I en un gel de metilcelulosa con una membrana de politetrafluoretileno en perros Beagle. La superficie del implante en contacto con hueso era de un $41,09 \%$ en los implantes tratados con factores 
de crecimiento y membrana y de un $23,16 \%$ utilizando la membrana sola (5).

Anitua usó el PRP en la preparación de emplazamientos para futuros implantes. En 20 pacientes trató los alvéolos de extracción con PRP o los dejó sin tratar para utilizarlos como controles. En los alvéolos a los que añadió PRP observó una mayor anchura ósea bucolingual/ palatina, una mayor densidad ósea y una cobertura tisular más rápida que en los controles (27).

Kassolis y cols. Han publicado recientemente los resultados de 14 injertos sinusales y 3 aumentos del reborde maxilar previos a la colocación de implantes, utilizando PRP con aloinjertos de hueso liofilizado (LifeNet). En los cortes histológicos observaron numerosas zonas de formación osteoide y hueso alrededor de las partículas de aloinjerto de hueso liofilizado, sin indicios de infiltrado celular inflamatorio $(27,9)$.

Whitman y cols. También han obtenido una adecuada consolidación del injerto particulado de hueso esponjoso y medular al utilizar PRP, ya que facilitaba la manipulación de los tejidos blandos durante la cirugía, evitando que estos causaran un desplazamiento inoportuno del injerto. También han utilizado PRP para subsanar perforaciones orales que han sido producidas durante la cirugía, así como en elevaciones de seno y para aumentar la anchura alveolar antes de la colocación de los implantes (25).

Martelli y cols. proponen la utilización del láser de Nd:YAG a baja potencia ofrece un efecto de descontaminación y de bioestimulación de los tejidos blandos y duros, siendo su acción superior a la de los agentes químicos más utilizados. Se aplica tras el legrado meticuloso de la zona contaminada obteniéndose unos resultados muy buenos (31).

Rodríguez et al llevaron a cabo elevaciones sinusales con la utilización de Bio-Oss y Plasma Rico en Plaquetas junto con la inserción de implantes dentales. Los resultados obtenidos fueron muy positivos (32).

Últimamente se han realizado estudios utilizando mini-cerdos debido a que al parecer son similares a los humanos en cuanto al recuento plaquetario, a los parámetros de coagulación y a la estructuración ósea:

- Gabory cols. Trataron de determinar los efectos de los FC liberados del PRP en relación al aumento de contacto implante-hueso. Además tratan de investigarlo en un hueso de Clase I, es decir formado principalmente por hueso cortical, pues hasta el momento no se habían hecho estudios en este tipo de hueso con un reconocido más bajo potencial de cicatrización. Los resultados obtenidos fueron positivos concluyendo que el PRP puede aumentar la anchura del hueso cortical alrededor del implante (33).

- En un estudio realizado por Wernery cols. Se investiga la influencia del PRP en la cicatrización temprana, al ser aplicado durante la inserción de los implantes dentales. Se obtiene como resultado que el PRP presenta u efecto dependiente del tiempo y del lugar de colocación en la cicatrización alrededor del implante, pues el mayor contacto implante -hueso se obtuvo durante las primeras semanas y en la zona de directa aplicación del concentrado plaquetario (34).

- En un experimento en cerdos llevado a cabo por Andreas y cols.Se pretendía evaluar microradiográficamente la osteointegración obtenida tras el tratamiento de los lechos implanterios con distintos métodos tópicos: PRP, Colloss o condensación. El efecto beneficioso del PRP tuvo lugar sobretodo durante la primera semana de cicatrización (35).

Otros estudios muestran unos resultados menos alentadores:

Stuart y cols. quisieron comprobar la utilidad del PRP junto con los injertos compuestos, es decir, aquellos formados por una variedad de aloinjertos, xenoinjertos, aloplastos y proteínas morfogenéticas óseas. Los resultados de mejora obtenidos no fueron ni clínica ni estadísticamente significativos. Aunque, aparte de cualquier efecto que pueda tener el PRP sobre la cicatrización de las heridas, la adición de PRP activado con trombina permitió mejorar considerablemente las propiedades de manipulación del material de injerto en partículas (27).

Shanaman y cols quisieron valorar el potencial del PRP junto con un aloinjerto óseo para aumentar la regeneración ósea en los defectos de la cresta alveolar con pérdidas verticales y horizontales previas a la colocación de implantes dentales. La adición de PRP no pareció aumentar la calidad y la cantidad de neoformación ósea, en comparación con la registrada en los estudios de ROG sin PRP (14).

Wironen y cols. Han comprobado que el PRP no tiene efectos osteoinductivos cuando se añade a una matriz de hueso desmineralizado introducida en bolsas creadas en los músculos rectos abdominales de ratas atímicas (27).

Gabory cols. Realizan elevaciones sinusales rellenando con Hidroxiapatita sola o en combinación con PRP simultáneamente a la inserción de los implantes dentales. Se vio que el PRP en combinación con Hidroxiapatita no mostraba resultados superiores frente a la utilización exclusiva de Hidroxiapatita (36). 
Otro tipo de material alogénico utilizado para elevaciones sinusales por Wiltfang y cols. es el TCP-b junto con PRP. Observan que la regeneración es menos extensa, pero que la reabsorción no está aumentada (37).

Dentro de las ventajas del uso del PRP destacan:

El PRP es el complemento ideal para los injertos, ya que sirve para compactar y retener el material de injerto, tanto autólogo como cualquier biomaterial, aportando estabilidad y adhesión. Además es un excelente osteoconductor y osteoinductor. Se trata de un material autólogo y por lo tanto con nulo efecto antigénico. No hay riesgo de contagio de ningún tipo de enfermedad y, por último, la fibrina autóloga obtenida con el PRP se puede utilizar a modo de membrana biológica para retener el injerto (38).

$\checkmark$ El PRP ayuda a obtener un efecto hemostático así como un aumento en la velocidad de cicatrización (9). Se ha utilizado para prevenir el sangrado tras la extracción dental en pacientes tratados con anticoagulantes orales sin emplear de modo coadyuvante heparina (39).

$\checkmark$ Con el PRP al mismo tiempo que se sella el alvéolo evitando su contaminación se proporciona una gran concentración de factores de crecimiento (3).

$\checkmark$ Se puede utilizar PRP en pacientes no candidatos a recibir donaciones de sangre como niños menores de 6 años, los ancianos u otros con determinadas condiciones sistémicas (25).

$\checkmark$ La incorporación de PRP en el protocolo para el tratamiento de fracasos implantarios acelera la cicatrización y maduración de los tejidos injertados (28).

El PRP resulta válido absolutamente para todos los pacientes, resultando imprescindible para pacientes de riesgo como fumadores, pues son dos mundos diferentes la epitelización de un fumador con o sin factores de crecimiento (40).

\section{RIESGOS CON EL USO DE PRP}

En un artículo realizado por el Dr. Martínez-González et al en la revista Medicina Oral de 2002, se postula una posible relación entre el uso de PRP y la aparición de tumores malignos. Se basan en que es conocido que en la carcinogénesis las sustancias promotoras van a actuar únicamente sobre el aumento de la proliferación celular en los clones de células inicialmente mutadas mediante la modificación de algunos procedimientos bioquímicos celulares.
Si no se promoviera la mitogénesis de esas células inicialmente mutadas, los mecanismos de control podrían desencadenar la muerte de esa célula alterada antes de que pudiera llegar a su diferenciación final. Así pues, los concentrados terapéuticos de factores de crecimiento podrían actuar, más que como iniciadores, como promotores en la carcinogénesis, favoreciendo la división y promoción de células previamente mutadas o "iniciadas" en la carcinogénesis.

Sin embargo, este fenómeno podría necesitar de dosis más continuadas en el tiempo que las que se aplican en la terapéutica del PRP, teniendo en cuenta que los factores de crecimiento extracelulares se degradan a los 7-10 días.

Otro factor a considerar es la capacidad antiapoptótica que se ha asignado a ciertos factores de crecimiento como el IGF y el VEGF.

En cualquier caso, la revisión de la literatura no ha permitido aportar evidencias científicas que relacionen la aplicación terapéutica de PRP con la transformación carcinomatosa de tejidos normales o displásicos, pero estos autores proponen una serie de medidas de prevención de los posibles riesgos:

- Realizar técnicas de obtención de PRP de una sola centrifugación, para obtener la mínima dosis efectiva.

- Evitar la utilización de PRP en pacientes con condiciones precancerosas orales ni en su proximidad.

- Evitar la aplicación de PRP en el "campo de cancerificación" de pacientes con exposición previa a carcinógenos 0 antecedentes de COCE (Carcinoma Oral de Células Escamosas) primario (22).

En la técnica para la utilización del PRP y la obtención del mismo en forma de gel se ha utilizado trombina tópica bovina, lo cual ha sido relacionado por varios autores con el desarrollo de anticuerpos contra el factor $\mathrm{V}$ y XI dando lugar a la aparición de coagulopatías mortales.

Además este fenómeno no parece ser un fenómeno dosis-dependiente $(2,38)$.

Landesber y cols proponen la adición de un gel de ITA en lugar de utilizar trombina bovina para evitar el riesgo de coagulopatías, pero no reflejan la composición química del producto, por lo que su uso no es recomendado $(4,41,42)$.

Por otro lado otros autores como Marx contraponen afirmando que el uso de PRP en cirugía oral y maxilo- 
facial utiliza trombina bovina para gelificar la preparación fuera del cuerpo, por lo que no entra en contacto directo con la circulación sistémica, y por lo tanto su utilización no presenta ningún riesgo (43).

Como conclusiones podemos decir lo siguiente:

1. Debido al aumento objetivo en la demanda de implantes dentales, es fundamental el esfuerzo por la búsqueda de nuevas técnicas que permitan resolver los casos clínicos más complicados.

2. El uso de los concentrados de plaquetas (PRP) ha resultado ser una innovación en el campo odontológico y, a medida que pasa el tiempo, cada vez son más los estudios experimentales y las mejoras realizadas sobre su utilización en cirugía oral.

3. Sería muy necesario la realización de convenios nacionales para regular el empleo de esta técnica. Asimismo, deberían establecerse las dosis mínimas necesarias para obtener el efecto terapéutico deseado.

4. Los estudios científicos muestran buenos resultados de la utilización de PRP en tejidos blandos, sin embargo, su utilización a nivel óseo no está tan clara.

5. Más estudios científicos son requeridos para descubrir la composición exacta del PRP obtenido, pues se sabe de la existencia de otros factores de crecimiento que aún no han sido reconocidos.

6. Por otro lado, y hasta que se hagan más estudios, conviene seguir las medidas para la prevención de complicaciones tumorales propuestas por el equipo del Dr. Martínez-González.

7. El cirujano, al igual que está al día de los últimos implantes que aparecen en el mercado, debe familiarizarse con el uso de los concentrados de plaquetas para incluirlos dentro de su propio arsenal terapéutico y, así, poder ofrecer a sus pacientes la mejor alternativa en cada situación.

\section{SUMMARY}

A review about using Platelet Rich Plasma in Implantology is presented. It begins with the basic concepts of Bone Guided Regeneration. Then, growth factors and its implication in Platelet Rich Plasma are described. We want to highlight the experiments with dental implants and PRP. It is also described the probably negative efects of PRP. Finally, we show the rewiev conclusions.

\section{KEY WORDS}

Platelet-rich-plasma, PRP, Growth Factors, Bone regeneration.

\section{BIBLIOGRAFÍA}

1. Bowen A. et al. Técnicas quirúrgicas avanzadas para la regeneración ósea en implantología. Gaceta Dental, 2002;128: 36-60.

2. Velilla M. et al. Recuerdo y actualización de las técnicas en Regeneración ósea para el práctico general. A propósito de dos casos. Gaceta Dental 2002; 127: 52-63.

3. Anitua E., Andia I. Valoración de la regeneración ósea en un modelo animal: utilización de Plasma Rico en Factores de Crecimiento (PRGF). Gaceta Dental, 2001; 123: 51-4.

4. Peñarrocha M., Sanchis J.M., Martínez J.M. Factores de crecimiento y proteínas que influyen en el crecimiento óseo: aplicaciones en implantología oral. Periodoncia, 2001; 11 (3): 205-16.

5. Serrano V., Casas A. Factores de crecimiento: ¿Un nuevo enfoque terapéutico? Periodoncia, 1997; 7 (2): 99-115.

6. Ibero I., Castro J. Factores de crecimiento y enfermedad periodontal. www.dentisnet.com, 2002. Revisiones.

7. Anitua Aldecoa E. Un nuevo enfoque en la regeneración ósea. Plasma rico en factores de crecimiento (PRGF). Puesta al Día publicaciones, SL. Vitoria, 2000.

8. Meraw S. et al. Treatment of Peri-Implant Defects With Combination Growth Factor Cement. J Periodontol, 2000; 71 (1): 8-12.

9. Nathan E., Rober B. Platelet-rich plasma. Clinical aplications in dentistry. JADA, 2002; 133: 1383-6.

10. Dugrillon A. et al. Autologous concentrated platelet-rich plasma (cPRP) for local application in bone regeneration. Int. J. Oral Maxillofac. Surg, 2002; 31:615-9.

11. Romo-Simón L. et al. Plasma rico en factores de crecimiento óseo en quistes maxilares y sobre implantes. Resultados preliminares. RCOE, 2001; 6: 48.

12. Anitua E. Plasma Rich in Growth Factors: Preliminary results os use in the preparation of future sites for implants. The Internatinal J. Of Oral and Maxillofac. Implants, 1999; 14: 529-35.

13. Marx R. et al. Platelet-rich plasma. Oral Surg Oral Med Oral Pathol Oral Radiol Endod, 1998; 85: 638-46.

14. Shanaman R. et al. Aumento localizado de la cresta utilizando ROG y plasma rico en plaquetas: casos clínicos. Int J Periodontics Restorative Dent, 2001; 21: 345-55. 
15. Anitua E. BTI Implant System: el primer sistema de implantes con superficie bioactiva. Maxillaris, 2001; diciembre: $38-43$.

16. Esra N. et al. Growth Factors Regulate Expresión of Mineral Associated Genes in Cementoblasts. J Periodontol, 2000; 71: 1591-600.

17. Cornelini R. et al.Vascular Endothelial Growth Factor and Microvessel Density around healthy and failing implants.Int J Oral Maxillofac Implants, 2001; 16: 389-93.

18. Sánchez A. et al. Is platelet-rich Plasma the Perfect Enhacement Factor? A current Review. Int J Oral Maxillofac Implants, 2003; 18: 93-103.

19. Lozada J. et al. Platelet - Rich Plasma Application in sinus graft surgery: Part I- background and processing techniques. Journal of Implantology, 2001; 27 (1): 38-42.

20. Lynch S. et al . The effects of short-term application of a combination of Platelet- Derived and Insulin-Like Growth Factors on periodontal wound healing. J Periodontol, 1991; 62: 458-67.

21. Lynch S. A combination of Platelet-Derived and InsulinLike growth factors enhances periodontal regeneration. J Clin Periodontol, 1989; 16: 545-8.

22. Lynch S. Effects of the Platelet-Derived Growth Factor/Insulin-Like Growth Factor-I combination on bone regeneration around titanium dental implants. Results of a pilot sttudy in Beagle Dogs. J Periodontol, 1991; 62: 710-6.

23. Martínez-González J.M. et al. ¿Existen riesgos al utilizar los concentrados de Plasma Rico en Plaquetas (PRP) de uso ambulatorio? Medicina Oral, 2002; 7: 375-90.

24. Tayapongsak P. et al. Autologous Fibrin Adhesive in Mandibular Reconstruction with Particulate Cancellous Bone and Marrow. J Oral Maxillofac Surg, 1994; 52: 161-5.

25. Whitman D. et al. Platelet Gel: An autologous alternative to Fibrin Glue with applications in oral and Maxillofacial surgery. J Oral Maxillofac Surg, 1997; 55: 1294-9.

26. Weibrich G. et al. Growth Factor levels in the Platelet Rich Plasma produced by 2 different methods: Curasan-type PRP Kit versus PCCS PRP System. Int J Oral Maxillofac Implants, 2002; 17: 184-190.

27. Froum S. et al. Efecto del plasma rico en plaquetas sobre el crecimiento óseo y la osteointegración en injertos de seno maxilar es seres humanos: tres informes de casos bilaterales. Int J Periodontics Restorative Dent, 2002; 22: 45-53.

28. Petrungaro P. Treatment of the infected Implant Site Using Platelet-Rich Plasma. Compendium, 2002; 23 (4): 363-76.

29. Kim S. et al. A comparative study of osseointegration of Avana Implants in a Desmineralized Freeze-Dried Bone alone or with Platelet-Rich Plasma.J Oral Maxillofac Surg, 2002; 60: 1018-25.
30. Kim S. et al. Use of Particulate Dentin-Plaster of Paris Combination with/without Platelet-Rich Plasma in the treatment of bone defects around implants. Int J Oral Maxillofac Implants, 2002; 17: 86-94.

31. Martelli et al. Aplicaciones clínicas del láser en implantología. Rev. Esp. Odontoestomtológica de Implantes, 2002; 10 (2): 60-5.

32. Rodríguez A. et al. Maxillary Sinus Augmentation with Deproteinated Bovine Bone and Platelet Rich Plasma with simultaneous insertion of endosseous implants. J Oral Maxillofac Surg, 2003; 61: 157-63.

33. Fuerst G. et al. Enhanced Bone-to- Implant Contact by Plateled-released Growth Factors in Mandibular Cortical Bone: A histomorphometric Study in minipigs. Int J Oral Maxillofac Implants, 2003; 18: 685-90.

34. Zechner W. et al. Influence of Platelet-rich Plasma on Osseous Healing of Dental Implants: $A$ histologic and Histomoorphometric Study in minipigs. Int J Oral Maxillofac Implants, 2003; 18: 15-22.

35. Andreas K. et al. Bone conditioning to enhance implant osseointegration: An experimental Study in Pigs. Int J Oral Maxillofac Implants, 2003; 18: 505-11.

36. Fuerst G. et al. Sinus grafting with autogenous plateletrich plasma and bovine hydroxyapatite: A histomorphometric study in minipigs. Clin. Oral Impl. Res., 2003; 14: 500-8.

37. Wiltfang J. et al. Sinus floor augmentation with B-tricalciumphosphate: does platelet-rich plasma promote its osseous integration and degradation?. Clin. Oral Impl. Res., 2003; 14: 213-8.

38. Anitua E. Expansión de cresta con osteotomos: Estado actual. Utilización de plasma rico en factores de crecimiento (P.R.G.F). Rev Esp Cirug Oral y Maxilofacial, 2001; 23: 158-62.

39. Della Valle A. et al. Prevention of postoperative bleeding in anticoagulated patients undergoing oral surgery: use of platelet-rich plasma gel. J Oral Maxillofac Surg, 2003; 61: 1275-8.

40. Anitua E. "La estimulación de la regeneración ósea y aceleración de la epitelización que provoca el PRFG es incuestionable". Gaceta Dental, 2001. Septiembre.

41. Landesberg R. et al. Risks of using Platelet Rich Plasma Gel .J Oral Maxillofac Surg, 1998; 56: 1116-7.

42. Landesberg R. et al. Quantification of Growth factor levels using a simplified method of Platelet-Rich Plasma Gel Preparation. J Oral Maxillofac Surg, 2000; 58: 297300.

43. Marxs R . Quantification of Growth factor levels using a simplified method of Platelet-Rich Plasma Gel Preparation. J Oral Maxillofac Surg, 2000; 58: 300-1. 